elongating, tends to form a spherical mass of tissue to which longitudinal growth is restored by washing the seeds in distilled water. In these cases growth is inhibited, but in certain others an inhibitory effect on germination itself may also be demonstrated. With prolonged exposure to the stimulant in the second phase, as indicated by the data presented above, germination tends to decrease, but here again washing the seeds before exposure to a higher temperature in the third phase frequently restores a high percentage germination to the sample.

The similarity between the effects of the two substances on the development of dormant organs, and on the germination of the seed of Striga lutea, suggests a corresponding similarity in the internal conditions of the dormant organs and of the parasite seed before exposure to the host stimulant. On the other hand, it must be emphasized that the similarity is not close, since many substances which break dormancy have no effect on the parasite seed. For example, while Thompson and Kossar found that thiosemicarbazide, thioacetamide, ammonium thiocyanate, and potassium thiocyanate were also effective in inducing. germina. tion in dormant lettuce, the same substances have no effect with the seed of Striga lutea.

R. BroWN.

M. EDWARDS.

Botany Department, The University,

Manchester.

'Brown, R., and Edwards, M., Ann. Bot., 8, 131 (1944).

${ }^{2}$ Denny, F., Bot. Gaz, 81, 297 (1926).

${ }^{3}$ Deuber, C. G., Science, 73, 320 (1931).

- Thompson, R. C., and Kossar, W. F., Plant Phys., 14, 567 (1939).

${ }^{5}$ Rayleigh, G. J., Science, 98, 538 (1943).

' Thompson, R. C., Science, 100, 131 (1944)

\section{Purification of Anterior Pituitary Corticotrophic Hormone}

IN the course of attempts to purify the corticotrophic hormone of the pituitary anterior lobe in the past few years, it was noted that the corticotrophic hormone shows far greater solubility in alcohol than other pituitary anterior lobe principles; it was also found to be more thermostable. Fresh pituitaries were therefore refluxed with ten times their weight of absolute ethyl alcohol (final alcohol concentration about 95 per cent) at $p \mathrm{H} 5$. Such extracts gave a far higher yield of corticotrophic activity than any other extraction method previously employed; but the most interesting fact found in connexion with the hot alcoholic extraction of the glands was that, on being filtered and cooled, the extract deposited a considerable amount of solid matter which quickly settled. The corticotrophic activity of both the supernatant fluid and of the deposit were estimated by the method of Reiss et al. ${ }^{1}$. A considerable amount of corticotrophic activity was found in the fluid, but it was of particular interest that the acetone-dried deposit contained an unexpectedly high amount of corticotrophic hormone. Other anterior pituitary biological activity could not be found.

The supernatant liquid yielded-after concentration in vacuo a further amount of solid material, which was also found to have a high degree of corticotrophic activity. We deduce, therefore, that the corticotrophic fraction is comparatively very soluble in warm alcohol, but sparingly soluble in cold alcohol.
The advantage of this method of purifying the corticotrophic principle would seem to be that an active corticotrophic hormone fraction is obtained which contains relatively little inert material or other anterior pituitary hormone.

The acetone-dried deposit can be completely dissolved in warm glacial acetic acid and such a solution forms, on cooling, a partly crystalline precipitate. The further fractionation of the partly crystalline material and the investigation of its biological activity are being carried out.

\section{Max Rerss.}

Y. M. L. GoldA.

Endocrinological Department,

Burden Neurological Institute, Bristol.

Feb. I6.

${ }^{1}$ Endokrin., 18, 1 (1936).

\section{Possible Synergistic Effect of Cod Liver Vitamin D on Synthetic Vitamin $D_{3}$}

Stott and Harris suggest" that "when synthetic vitamin $D_{3}$ is blended with cod liver oil the assay, in international units, is higher than the calculated figure", and cite two typical results of tests using eighteen pairs of rats in each assay. They also cite the average figures for thirteen blends of cod liver oil; but there is some ambiguity as to whether the averages also apply to the calculated and ascertained potencies of the resulting mixtures.

While their conclusion is of course not impossible, prudence would suggest that if it is to be generally accepted, further and more precise data should be provided. Although it is clear from the context that the biological tests used depend on the cure of experimental rickets in the rat, the authors do not mention the precise method used.

Assuming either of the British Pharmacopoeia methods to have been used, the fiducial limits at $p=0.99$, using ten pairs of rats, may be as wide as 49-215 per cent', but much will obviously depend on the precision attained in Stott and Harris's own laboratory. No indication of this is given. The point is of especial importance because no fewer than three biological assays enter into each comparison, namely, assays of the original oil, the vitamin $\mathrm{D}_{3}$ concentrate and the final mixture.

With fiducial limits of the order of those cited in the British Pharmacopoeia--which may be even wider in the tests under discussion - it is obvious that many repetitions would be needed before an interesting possibility could be regarded as a statistically significant finding.

Further information should also be provided as to the genesis of the figures for the "synthetic vitamin $\mathrm{D}_{3}$ ". This is presumably the commercial product which is, strictly speaking, not synthetic at all and which is normally assayed and sold -as required by law-in terms of B.S.I. units, not in terms of international units. Stott and Harris's use of the term 'international units' in this context presumably implies that they have re-assayed it on rats before use. If so, it would be of interest to see a comparison between the assay in B.S.I. units, on which the concentrate was purchased, and their assay in international units. If, on the other hand, no re-assay has been carried out, then the use of the term 'international units' is not justified. 